\title{
A Versatile Strategy for Shish-Kebab-like Multi-heterostructured Chalcogenides and Enhanced Photocatalytic Hydrogen Evolution
}

\author{
Jianqiang $\mathrm{Hu},{ }^{\dagger}$ Aili Liu, ${ }^{\dagger}$ Huile Jin, ${ }^{\dagger}$ Dekun Ma, ${ }^{\dagger}$ Dewu Yin, ${ }^{\dagger}$ Pengsheng Ling, ${ }^{\dagger}$ Shun Wang, ${ }^{*}{ }^{\dagger}$ \\ Zhiqun Lin, ${ }^{*}+\frac{}{*}$ and Jichang Wang, ${ }^{*}$, \\ ${ }^{\dagger}$ College of Chemistry and Materials Engineering, Wenzhou University, Wenzhou, Zhejiang 325035, China \\ ${ }^{\star}$ School of Materials Science and Engineering, Georgia Institute of Technology, Atlanta, Georgia 30332-0245, United States \\ ${ }^{\S}$ Department of Chemistry and Biochemistry, University of Windsor, Windsor, ON, Canada N9B 3P4
}

\section{Supporting Information}

\begin{abstract}
A series of multi-heterostructured metal chalcogenides (CdS-Te, NiS/CdS-Te, and $\mathrm{MoS}_{2} / \mathrm{CdS}-\mathrm{Te}$ ) with a surprising shish-kebab-like structure have been synthesized via a one-step microwave-assisted pyrolysis of dithiocarbamate precursors in ethylene glycol. Subsequently, CdS-Te composites were exploited as a self-sacrificial template to craft various CdS-Te@(Pt, Pd) multiheterostructures. Highly uniform dispersion and intimate interactions between $\mathrm{CdS}$ and multicomponent cocatalysts, together with improved separation of photogenerated carriers due to the presence of Te nanotubes (NTs) and trace CdTe, enable CdSbased heterostructured photocatalysts to exhibit greatly enhanced

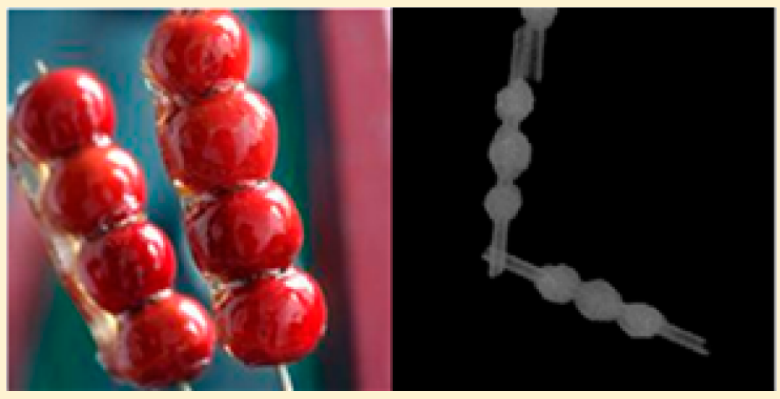
efficiency and stability in the photocatalytic production of $\mathrm{H}_{2}$. Thorough morphological characterizations revealed that the growth of metal sulfide/Te heterostructures originates from the growth of Te tubes, which is likely governed by diffusion-limited depletion of the Te precursor and the dissolution-crystallization process of Te seeds followed by the formation of metal sulfide kebabs.
\end{abstract}

\section{INTRODUCTION}

Nanostructured metal chalcogenides (MCs) have garnered considerable attention because of their wide range of application in various areas including organic catalysis, environmental protection, and energy storage and conversion, to name a few. ${ }^{1}$ To explore and subsequently utilize synergetic coupling effects between MCs and other functional materials, there are increasing efforts to create MC-based heterostructures, in particular those with low or negligible energy barrier at their interface, to greatly facilitate the charge transfer. ${ }^{2} \mathrm{MC}-$ noble metal, ${ }^{3} \mathrm{MC}-$ metalloid, ${ }^{4} \mathrm{MC}-$ metal oxide, ${ }^{5} \mathrm{MC}-$ carbon, ${ }^{6}$ and $\mathrm{MC}-\mathrm{MC}^{7}$ heterostructures have been derived from gas-, liquid-, or solid-phase synthesis over the past few years. However, several key issues in the fabrication and application of MC-based heterostructured materials remain to be addressed. First, the synthesis of multicomponent heterostructures has so far relied on structure-directing templates, in which either a soft or hard template is employed to provide the needed heterostructure. ${ }^{8}$ The subsequent removal of the template not only complicates the procedure but also increases the fabrication cost, which may seriously restrict their large-scale production. In this context, it is of key importance to develop facile, scalable, energy-saving, and environmentally benign synthetic routes to MC-based heterostructured materials. ${ }^{1,9}$ Second, the performance of MC-based heterostructured materials reported to date still cannot meet the requirements for practical applications. ${ }^{10}$ For example, the activity and stability of MC-based heterostructured materials are still inferior to those of commercially available noble metal (Pt or $\mathrm{Pt}$ )-based catalysts for the oxygen reduction reaction (ORR), hydrogen evolution reaction (HER), and oxygen evolution reaction (OER). ${ }^{3 a, b}$ The capacity, energy density, coulombic efficiency, and cycling life of MC-based energy storage devices are still comparatively low and need to be greatly enhanced in order to reach a large-scale market adoption. ${ }^{11}$ Third, only a few approaches can provide good control over the microstructure and a uniform distribution of different components within the multi-heterostructured MC materials. ${ }^{12}$ Clearly, in this context, much attention needs to be concentrated on the design and development of multicomponent or new heterostructured MC-based materials; this remains a grand challenge. ${ }^{1 \mathrm{a}, 13}$

Herein we report a versatile strategy for one-step crafting of two-component (CdS-Te), three-component (NiS/CdS-Te, $\mathrm{MoS}_{2} / \mathrm{CdS}-\mathrm{Te}$, CdS-Te@Pd, and CdS-Te@Pt), and fourcomponent (CdS-Te@(Pt,Pd)) chalcogenide heterostructures. All of the resulting materials possess a surprising shish-kebablike structure in which a Te tube acts as the shish and MCs form the kebabs, dispensing with the need for the use of

Received: May 8, 2015

Published: August 13, 2015 
templates. In this work, the selection of Te as a constituent was motivated by its unique chemical and physical properties. Specifically, bulk $\mathrm{Te}$ shows metallic behavior at room temperature. The good electrical transport properties of Te nanotubes (NTs) have also been reported. ${ }^{14}$ The combination of Te NTs with CdS facilitates the separation of photogenerated electrons and holes, thus enhancing the photocatalytic activity and photostability of CdS. More importantly, Te can be used as a self-sacrificial template to form a series of new heterostructures while retaining the morphology. ${ }^{15}$ Subsequently, the shish-kebab-like MC heterostructures were exploited in photocatalytic water splitting to produce hydrogen. The uniform dispersion and intimate connection between CdS and the cocatalysts greatly enhance the efficiency and stability of the shish-kebab-like photocatalysts, opening a new avenue for the development of intimate multicomponent heterostructures for a wide variety of applications.

\section{RESULTS AND DISCUSSION}

Shish-Kebab-like CdS-Te Heterostructures. Figure 1a displays a scaning electron microscopy (SEM) image of asprepared CdS-Te heterostructures resembling a shish-kebablike structure. ${ }^{16}$ A high-magnification SEM image reveals that three microspheres (i.e., kebabs) are connected by a shish (Figure 1a inset) and that the shish is tubular-like with a
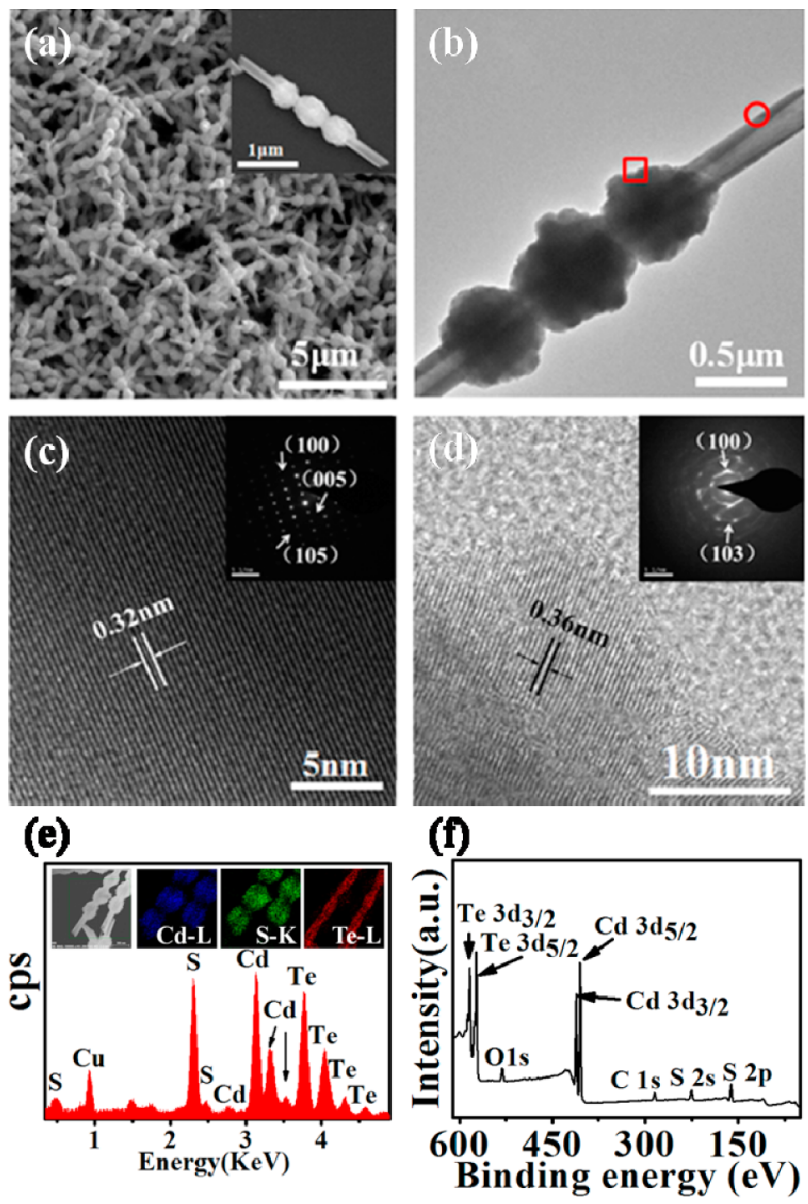

Figure 1. CdS-Te sample characterized by (a) SEM, (b) TEM, (c, d) HRTEM of the areas marked with (c) the red circle and (d) the red square in (b) (the insets show the corresponding SAED patterns), (e) EDX (the inset shows the STEM-EDX elemental maps), and (f) XPS. smooth surface, whereas the kebabs have a rough surface. The $\mathrm{X}$-ray diffraction (XRD) pattern shows that the products consist of hexagonal-phase Te (JCPDS no. 65-3370) and CdS (JCPDS no. 41-1049) (Figure $S 1$ in the Supporting Information). The above CdS-Te sample was prepared by adding $0.069 \mathrm{mmol}$ of diethyldithiocarbamatotellurium(IV) (TDEC), $0.122 \mathrm{mmol}$ of diethyldithiocarbamatocadmium(II) (CED), and $30.0 \mathrm{~mL}$ of ethylene glycol to a $50 \mathrm{~mL}$ roundbottom flask. After that, the suspension was microwave-heated to $90{ }^{\circ} \mathrm{C}$ at a power setting of $200 \mathrm{~W}$, which required about 1 $\mathrm{min}$. The solution was kept under heating for a total of $5 \mathrm{~min}$, during which it turned from yellow to clear at about $3 \mathrm{~min}$. Then the solution temperature was raised to $160{ }^{\circ} \mathrm{C}$ at a power setting of $500 \mathrm{~W}$, which needed about $1 \mathrm{~min}$ to reach. At a reaction time of about $6 \mathrm{~min}$, the suspension solution turned dark green, indicating the formation of CdS-Te heterostructures. The resulting solid products were collected by centrifugation at $10000 \mathrm{rpm}$ for $3 \mathrm{~min}$, washed with deionized water and ethanol several times, and finally dried in vacuum at $60{ }^{\circ} \mathrm{C}$ for $12 \mathrm{~h}$.

Transmission electron microscopy (TEM) measurements show that the as-prepared CdS-Te heterostructures possess a hollow tubular structure (Figure $1 \mathrm{~b}$ ); however, the tube center is solid, as reported in the literature, showing an internal structure that looks like a wedge. ${ }^{17}$ These nanotubes range from 300 to $320 \mathrm{~nm}$ in outer diameter and from 4.8 to $5.4 \mu \mathrm{m}$ in length. The HRTEM image in Figure 1c, taken from the local area marked with a ring in Figure $1 b$, shows that the lattice fringes are structurally uniform with a spacing of $0.320 \mathrm{~nm}$. Such lattice fringes are in good agreement with the $d$ value of the (011) plane of hexagonal Te. The selected-area electron diffraction (SAED) pattern in the Figure 1c inset clearly suggests that the nanotube is made up of a single Te crystal. ${ }^{17 b}$ The HRTEM image in Figure 1d was taken from the area labeled with a square in Figure $1 \mathrm{~b}$ and shows a lattice spacing of $0.360 \mathrm{~nm}$, which corresponds to the (100) planes of hexagonal wurtzite-structured CdS. ${ }^{5 \mathrm{~b}}$ The SAED pattern (Figure $1 \mathrm{~d}$ inset) indicates that the $\mathrm{CdS}$ kebab is polycrystalline. The energydispersive X-ray (EDX) spectrum in Figure 1e confirms the presence of $\mathrm{Cd}, \mathrm{S}$, and Te elements. The $\mathrm{Cu}$ peak originates from the copper TEM grid used as a sample holder. The inset in Figure le shows the EDX elemental maps of the CdS-Te sample, signifying that $\mathrm{Cd}$ (blue) and $\mathrm{S}$ (green) are evenly distributed within the kebabs and Te (red) is evenly distributed in the nanotube.

The chemical compositions and electronic structures of the CdS-Te heterostructures were analyzed by X-ray photoelectron spectroscopy (XPS). From the peaks in the survey spectrum in Figure 1f, it is clear that $\mathrm{Cd}, \mathrm{S}$, and Te exist in the heterostructure. The high-resolution XPS spectrum of the Cd $3 \mathrm{~d}$ orbital region (Figure S2a) displays the binding energies of $\mathrm{Cd} 3 \mathrm{~d}_{3 / 2}$ and $\mathrm{Cd} 3 \mathrm{~d}_{5 / 2}$ at 411.72 and $404.96 \mathrm{eV}$, respectively. The splitting energy of $6.76 \mathrm{eV}$ between $\mathrm{Cd} 3 \mathrm{~d}_{3 / 2}$ and $\mathrm{Cd} 3 \mathrm{~d}_{5 / 2}$ is a signature of $\mathrm{Cd}^{2+}$. The binding energies of $\mathrm{S} 2 \mathrm{p}_{3 / 2}$ and $\mathrm{S}$ $2 \mathrm{p}_{1 / 2}$ in the sample are observed at approximately 162.41 and $161.2 \mathrm{eV}$, respectively (Figure S2b), in good agreement with the reference values for $\mathrm{S}^{2-}$. 583.53 and $573.2 \mathrm{eV}$ (Figure S2c) can be assigned to $3 \mathrm{~d}_{3 / 2}$ and $3 \mathrm{~d}_{5 / 2}$ of elemental $\mathrm{Te}$ according to the reference values in the literature. ${ }^{19}$ The fitting of the Te energy peaks also suggests the existence of $\mathrm{Te}^{2-}$, which is indicative of the possible formation of CdTe at the interface of the microspheres and the Te tube. 
Figure 2a shows that the one-step-synthesized NiS/CdS-Te heterostructures also resemble shish-kebabs, like the CdS-Te
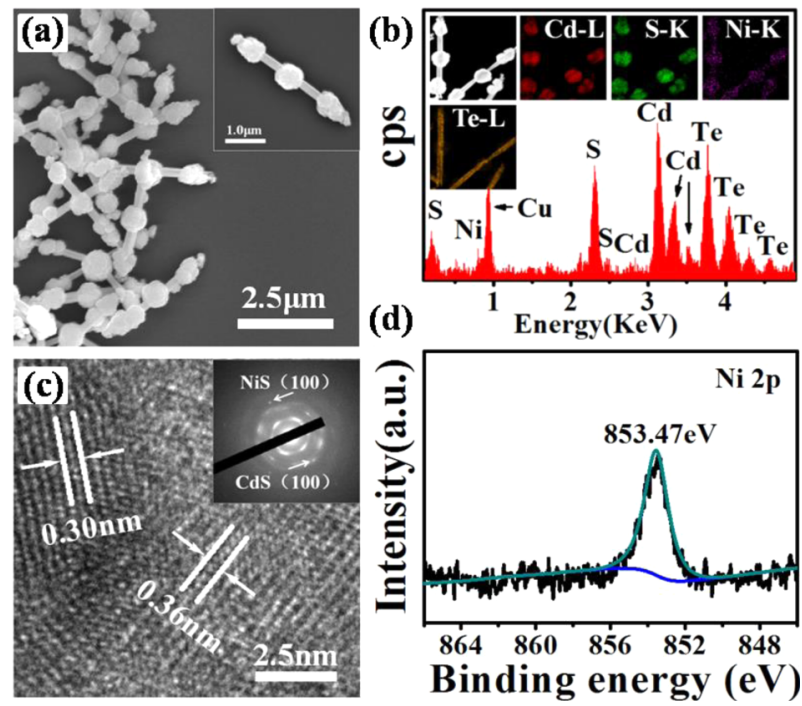

Figure 2. NiS/CdS-Te sample characterized with (a) SEM, (b) EDX (the inset shows the STEM-EDX elemental maps), (c) HRTEM (the inset shows the corresponding SAED pattern), and (d) high-resolution Ni 2p XPS.

heterostructures described above. The SEM image in the Figure $2 \mathrm{a}$ inset indicates that the three microspheres (i.e., kebabs) have a rough surface and are separated from each other on a smooth tube. The EDX spectrum verifies the presence of $\mathrm{Cd}, \mathrm{S}, \mathrm{Ni}$, and Te. The EDX elemental maps for the NiS/CdS-Te sample in Figure $2 \mathrm{~b}$ show that $\mathrm{Cd}$ (red), $\mathrm{S}$ (green), and $\mathrm{Ni}$ (purple) are uniformly distributed in the spheres and that Te (gold) is evenly distributed in the nanotube. The HRTEM image in Figure $2 c$, taken at an area around the sphere, displays that there are two types of lattice distance, 0.360 and $0.300 \mathrm{~nm}$, corresponding to the (100) planes of hexagonal wurtzitestructured CdS and the (100) planes of NiS, respectively. The SAED pattern (Figure $2 \mathrm{c}$ inset) indicates that the NiS/CdS kebabs are polycrystalline. This is consistent with the observation that there is no apparent NiS peak in the XRD pattern of $\mathrm{NiS} / \mathrm{CdS}-\mathrm{Te}$ heterostructures with relatively low amounts of NiS (Figure S3). The binding energy of Ni $2 \mathrm{p}$ in the high-resolution XPS spectrum presented in Figure $2 \mathrm{~d}$ is $853.47 \mathrm{eV}$, which is in good agreement with the reference value for NiS. ${ }^{20}$ XPS spectra of the NiS/CdS-Te sample and the highresolution XPS spectra of Cd 3d, S 2p, and Te $3 \mathrm{~d}$ are provided in Figure S4. The fitting of Te energy peaks indicates the existence of $\mathrm{Te}^{2-}$, which suggests the possible formation of $\mathrm{CdTe}$ at the interface of microspheres and the Te tube.

Through the same one-pot pyrolysis, $\mathrm{MoS}_{2} / \mathrm{CdS}-\mathrm{Te}$ heterostructures were also prepared. The SEM image in Figure 3a shows the same shish-kebab-like microstructure. The TEM image in Figure $3 \mathrm{~b}$ reveals that similar to the CdS-Te heterostructure, the shish has a hollow tubular structure with the three kebabs in close contact. The HRTEM image in Figure $3 c$, taken from the area marked with a square in Figure $3 b$, shows lattice constants of 0.301 and $0.360 \mathrm{~nm}$, corresponding to the (006) planes of $\mathrm{MoS}_{2}$ and the (100) planes of hexagonal wurtzite-structured $\mathrm{CdS}$, respectively. The EDX elemental maps (Figure 3d) and XPS measurements (Figure S5f) also strongly support on the formation of $\mathrm{MoS}_{2}$ in the as-prepared
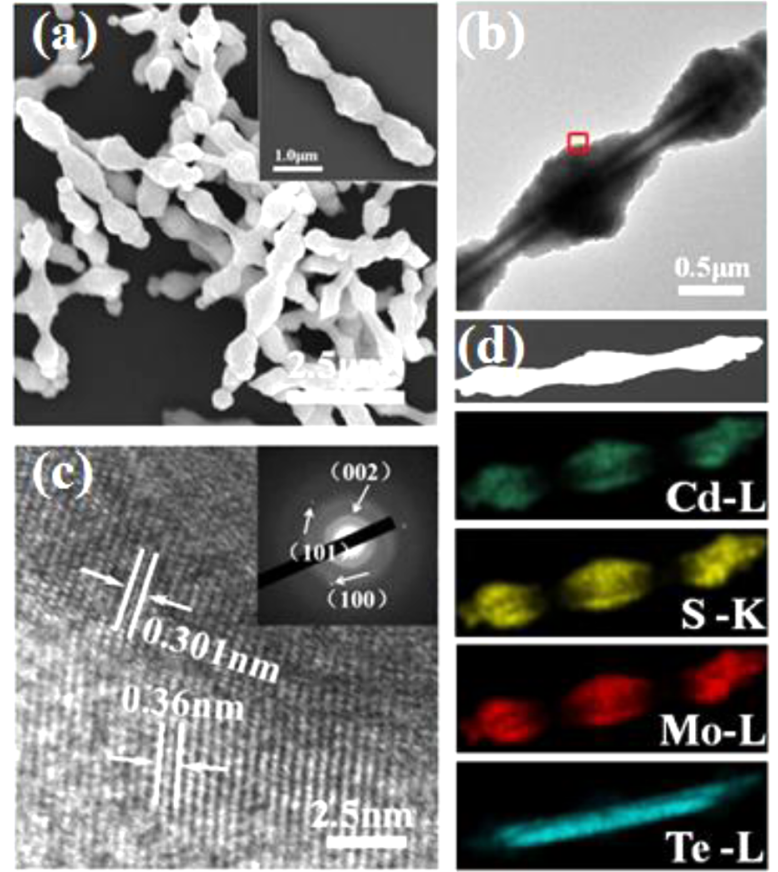

Figure 3. $\mathrm{MoS}_{2} / \mathrm{CdS}$-Te sample characterized with (a) SEM, (b) TEM, (c) HRTEM of the area marked with a square in (b) (the inset shows the corresponding SAED pattern), and (d) STEM-EDX elemental mapping, showing the Cd (green), S (yellow), Mo (red), and $\mathrm{Te}$ (turquoise).

heterostructure. The SAED pattern (Figure $3 \mathrm{c}$ inset) suggests a polycrystalline ring. The EDX elemental maps of the $\mathrm{MoS}_{2} /$ CdS-Te sample presented in Figure 3d show that Cd (green), S (yellow), and Mo (red) are uniformly distributed in the three microspheres, whereas Te (turquoise) is evenly distributed in the nanotube. The survey XPS spectrum and high-resolution XPS spectra further confirm the presence of $\mathrm{Cd}, \mathrm{S}, \mathrm{Mo}$, and Te in the $\mathrm{MoS}_{2} / \mathrm{CdS}$-Te heterostructure (Figure S5b). Similarly, fitting of the Te energy peaks signifies the existence of $\mathrm{Te}^{2-}$, suggesting the possible formation of $\mathrm{CdTe}$ at the interface.

In addition to the direct synthesis of MCs described above, the as-prepared CdS-Te was also exploited as a template to craft other new heterostructures, particularly CdS-Te@Pt,Pd heterostructures, which are expected to exhibit high catalytic activity due to the presence of the noble metals $\mathrm{Pt}$ and $\mathrm{Pd}$. The morphology of CdS-Te@Pt,Pd heterostructures shown in Figure $4 \mathrm{a}$ appears essentially identical to that seen for the CdS-Te heterostructures in Figure 1a, suggesting that the CdS$\mathrm{Te}$ heterostructures remain intact after their reaction with $\mathrm{H}_{2} \mathrm{PtCl}_{6}$ and $\mathrm{PdCl}_{2}$ for $4 \mathrm{~h}$ at $90{ }^{\circ} \mathrm{C}$. In comparison with that observed in Figure $1 \mathrm{~b}$, the surfaces of the Te tube (i.e., the shish) and the microspheres (i.e., the kebabs) become rougher, suggesting that new materials have been deposited onto the CdS-Te heterostructures. The close-up of the area marked with a red circle in Figure $4 \mathrm{~b}$ shows that the lattice fringes are no longer structurally uniform, where a spacing of $0.320 \mathrm{~nm}$ was observed, corresponding to the (011) planes of hexagonal $\mathrm{Te}$ (Figure 4c). The lattice fringe of ca. $0.227 \mathrm{~nm}$ for Pt and the lattice fringe of ca. $0.225 \mathrm{~nm}$ for Pd were also observed on the Te surface, implying that $\mathrm{Pt}$ and $\mathrm{Pd}$ were successfully loaded onto the Te tube.

The HRTEM image in Figure 4d, taken from the area marked with a square in Figure $4 b$, shows a lattice distance of $0.360 \mathrm{~nm}$, which corresponds to the (100) planes of hexagonal 

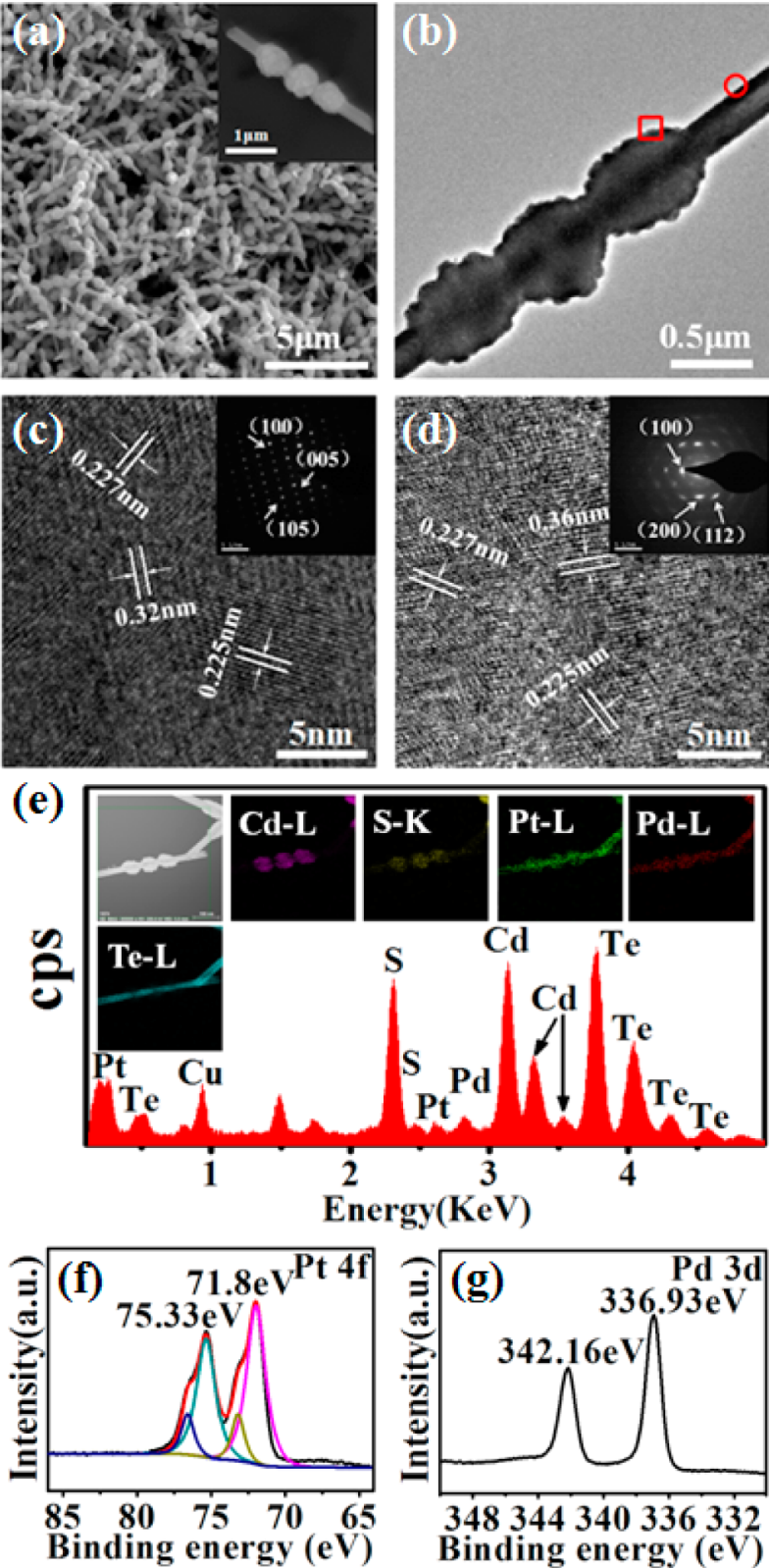

Figure 4. CdS-Te@Pt,Pd sample characterized with (a) SEM, (b) TEM, (c, d) HRTEM of the areas marked with (c) the red circle and (d) the red square in (b) (the insets show the corresponding SAED patterns), (e) EDX (the inset shows the STEM-EDX elemental maps for Cd (purple), S (yellow), Pt (green), Pd (red), and Te (turquoise)), and (f, g) high-resolution XPS for (f) Pt $4 \mathrm{f}$ and (g) Pd 3d.

wurtzite-structured CdS. The lattice fringe of ca. $0.227 \mathrm{~nm}$ for $\mathrm{Pt}$ and the lattice fringe of ca. $0.225 \mathrm{~nm}$ for Pd were also observed, implying that Pt and Pd were successfully loaded onto the spheres as well. Clearly, these results suggest that our synthetic approach can be employed to produce a large number of CdS-Te composites with a uniform heterostructure. The EDX spectrum in Figure 4e confirms the presence of $\mathrm{Cd}, \mathrm{S}, \mathrm{Te}$, $\mathrm{Pt}$, and Pd. The EDX elemental maps show evenly distributed Cd (purple), S (yellow), Pt (green), and Pd (red) in the kebabs and uniformly distributed Te (turquoise) in the nanotube (Figure $4 \mathrm{e}$ inset). According to the peaks in the survey spectrum in Figure S6a, it is obvious that $\mathrm{Cd}, \mathrm{S}, \mathrm{Te}, \mathrm{Pt}$, and $\mathrm{Pd}$ all exist in the CdS-Te@Pt,Pd heterostructure. In addition, the high-resolution $\mathrm{Pt} 4 \mathrm{f}$ spectrum in Figure $4 \mathrm{f}$ shows that $\mathrm{Pt} 4 \mathrm{f}_{7 / 2}$ and $\mathrm{Pt} 4 \mathrm{f}_{5 / 2}$ peaks are located at 71.8 and $75.33 \mathrm{eV}$, respectively, signifying that $\mathrm{H}_{2} \mathrm{PtCl}_{6}$ has been successfully reduced to metallic $\mathrm{Pt}^{21}$ As displayed in Figure $4 \mathrm{~g}$, the binding energies of 336.93 and $342.16 \mathrm{eV}$ are indicative of metallic $\mathrm{Pd}$, corresponding to the $3 \mathrm{~d}_{5 / 2}$ and $3 \mathrm{~d}_{3 / 2}$ peaks of $\mathrm{Pd}^{0}$, respectively. ${ }^{22}$ Figure S6 presents XPS spectra of the CdSTe@Pt,Pd sample and high-resolution XPS spectra of Cd 3d, S $2 \mathrm{p}$, and Te $3 \mathrm{~d}$. The ability to detect $S$ by XPS implies that the thickness of the boundary between the $\mathrm{Pt}$ and $\mathrm{Pd}$ cores and the outer edge of the CdS shell is likely no more than $10 \mathrm{~nm}$. Taken together, the results of SEM, TEM, EDX, XRD, and XPS measurements collectively substantiate the formation of CdSTe@Pt,Pd. The above procedure has also been exploited to prepare shish-kebab-like CdS-Te@Pt and CdS-Te@Pd heterostructures, and the related characterizations are presented in Figures S7-S12.

Figure 5 presents the diffuse-reflectance UV-vis absorption spectra of CdS, Te, CdS-Te, NiS/CdS-Te, $\mathrm{MoS}_{2} / \mathrm{CdS}-\mathrm{Te}$, and

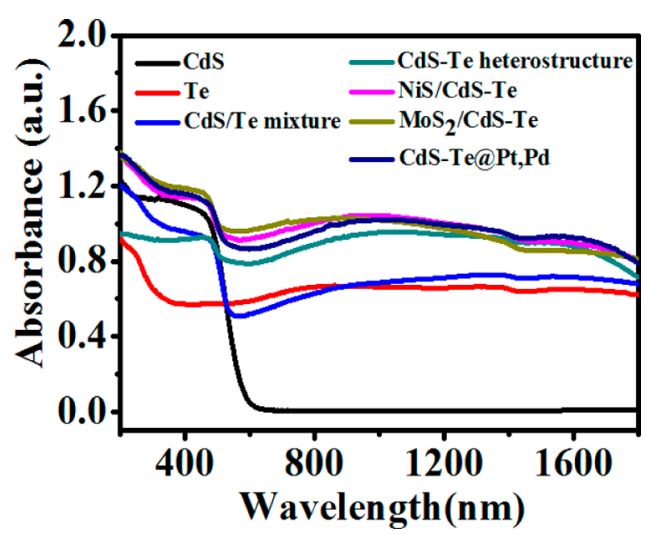

Figure 5. UV-vis absorption spectra of $\mathrm{CdS}, \mathrm{Te}, \mathrm{CdS} / \mathrm{Te}$ mixture, CdS-Te, NiS/CdS-Te, $\mathrm{MoS}_{2} / \mathrm{CdS}-\mathrm{Te}$, and CdS-Te@Pt,Pd samples.

CdS-Te@Pt,Pd heterostructures and a mixture of CdS and Te. Compared with the pure CdS sample, the CdS-Te@Pt,Pd heterostructures exhibit the most enhanced light absorption intensity in the range of 500-1400 $\mathrm{nm}$. The improved light absorption in the visible region suggests that CdS-Te@Pt,Pd heterostructures may enable higher light-driven photocatalytic activity than that of pure $\mathrm{CdS}$ nanoparticles for given reactions.

Growth Mechanism of the Shish-Kebab-like Heterostructures. We next turned our attention to the formation mechanism of shish-kebab-like CdS-Te heterostructures by scrutinizing their intermediate growth process at 6.0, 6.25, 6.5, $6.75,7.0$, and $10.0 \mathrm{~min}$ (Figure 6). The structure synthesized for 6.0 min contained mainly irregular particles (Figure 6a), which consisted of elemental $\mathrm{Te}$, undissolved $\mathrm{CED}$ (i.e., the precursor of the Cd and S sources), and TDEC (Figures S13 and S14). After reaction for $6.25 \mathrm{~min}$, Te nanotubes with lengths of $1-2 \mu \mathrm{m}$ emerged, signifying that Te nanotubes were formed first in the solution (Figure 6b). Similar to the scenario seen in the study by Xia and co-workers, ${ }^{17 a}$ in the present study the Te tubes in the synthesized CdS-Te composites have a cylindrical solid in the middle. Such a morphology suggests that the concentration depletion mechanism applies to the formation of the Te tubes. The rapid formation of a large quantity of elemental Te leads to the formation of many seeds through homogeneous nucleation. Immediately after the nucleation, when new Te atoms are produced in the solution, the circumferential edges of each seed preferentially grow 

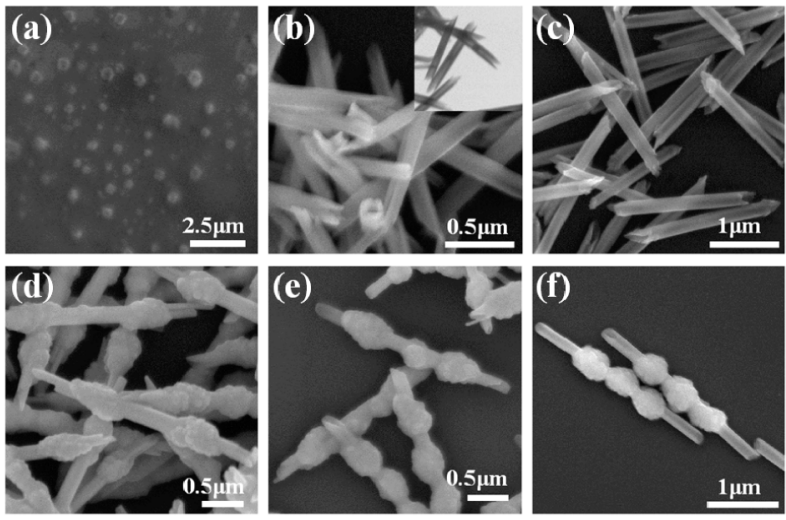

(g)

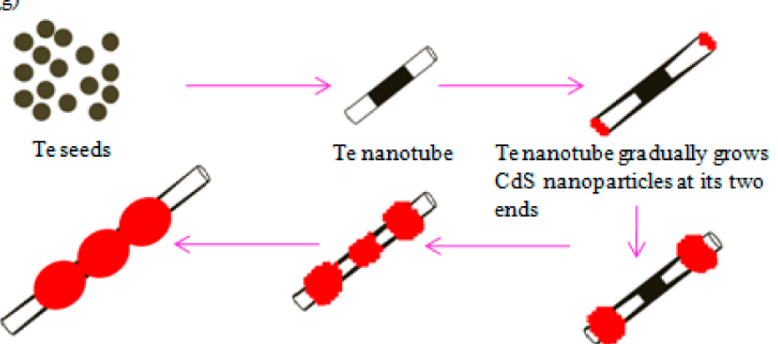

Figure 6. $(a-f)$ SEM images of CdS-Te heterostructures prepared at reaction times of (a) $6 \mathrm{~min}$, (b) $6.25 \mathrm{~min}$, (c) $6.5 \mathrm{~min}$, (d) $6.75 \mathrm{~min}$, (e) $7.0 \mathrm{~min}$, and (f) $10.0 \mathrm{~min}$. (g) Growth scheme for the shish-kebablike CdS-Te heterostructures.

because these sites have relatively higher free energies than other sites on the surface. Once the crystal growth begins, mass transport to the growing regions leads to undersaturation (or complete depletion) of Te in the central portion of the growing faces (i.e., the (001) planes) of each seed, eventually resulting in the formation of nanotubes possessing well-defined hollow interiors.

In the present study, the internal structure of these nanotubes looks like a wedge near the seed (i.e., the center), implying that nucleation-dissolution-recrystallization might have taken place as well. ${ }^{17}$ At $6.5 \mathrm{~min}$ (Figure 6c), the Te nanotubes grew to over $3 \mu \mathrm{m}$, accompanied by the emergence of a few irregular particles on those tubes. EDX analysis of these irregular particles revealed the chemical composition of $\mathrm{CdS}$. According to previous reports, ${ }^{23} \mathrm{CdS}$ nanocrystals, especially nanorods, have preferential (002) crystal growth. We note that the (001) lattice of the Te crystal and the (002) lattice of the CdS crystal match well. When the reaction proceeded for 6.75 min (Figure 6d), the irregular particles grown on the Te nanotubes became spherical. Meanwhile, the Te tube continued to grow, leading to a scene in which the two $\mathrm{CdS}$ beads were developed in the middle of the tube. With a further increase in the reaction time to $7.0 \mathrm{~min}$ (Figure 6e), spherical $\mathrm{CdS}$ was also developed in the center of $\mathrm{Te}$ nanotubes, indicating that the development of two CdS spheres made the section of Te tube between the two spheres suitable for CdS nucleation. Finally, the shish-kebab-like heterostructure was fully developed after $10 \mathrm{~min}$ (Figure 6f). On the basis of the above observations, a possible growth model is proposed in Figure $6 \mathrm{~g}$. At the beginning of the reaction, Te atoms are produced and aggregate to form Te nanotubes with an axial direction along the (001) faces. Subsequently, CED is thermally decomposed to form $\mathrm{CdS}$. The presence of many defects at the two ends of the Te tube as well as the lattice match between $\mathrm{Te}$ and $\mathrm{CdS}$ favors
$\mathrm{CdS}$ deposition there. In other words, the nucleation of $\mathrm{CdS}$ is preferentially initiated at the two ends of the Te nanotube. As the reaction progresses, $\mathrm{CdS}$ particles grow to become spherical, which is accompanied by continuous growth of the Te tube. With a further increase in reaction time, a CdS sphere is also grown at the center of the Te nanotube, thus leading to the formation of shish-kebab-like heterostructure.

We also conducted time-dependent experiments to understand the growth process of NiS/CdS-Te and $\mathrm{MoS}_{2} / \mathrm{CdS}-\mathrm{Te}$ heterostructures. Characterization (see Figures S15 and S16) indicated that the overall process is the same as for CdS-Te system, in which Te tubes are formed first, followed by nucleation of $\mathrm{CdS} / \mathrm{NiS}$ particles at the free ends of the Te tube. More time-dependent experiments are required in order to elucidate the formation mechanism of this interesting shishkebab microstructure, especially why most structures contain exactly three rather than, for example, four beads and why the CdS did not prevent further growth of the Te NTs.

Photocatalytic Activity. Subsequently, the as-prepared MCs were employed to catalyze the water splitting reaction. ${ }^{20,24}$ In this study, the photocatalytic $\mathrm{H}_{2}$ production used lactic acid as the sacrificial reagent. Control experiments indicated that no appreciable $\mathrm{H}_{2}$ production was detected in the absence of either irradiation or the MC. Figure 7a compares the
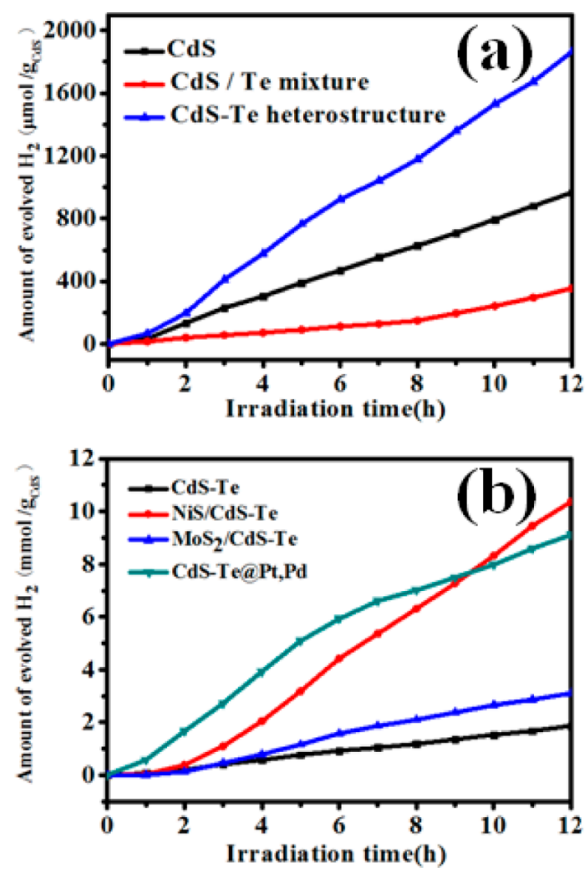

Figure 7. Comparison of the photocatalytic activities of (a) CdS, a $\mathrm{CdS} / \mathrm{Te}$ mixture, and CdS-Te heterostructures and (b) CdS-Te, NiS/ $\mathrm{CdS}-\mathrm{Te}, \mathrm{MoS}_{2} / \mathrm{CdS}-\mathrm{Te}$, and CdS-Te@Pt,Pd in a lactic acid aqueous solution under visible-light illumination.

photocatalytic $\mathrm{H}_{2}$ production rates of CdS, a mixture of $\mathrm{CdS}$ and $\mathrm{Te}, \mathrm{CdS}$-Te heterostructures under illumination at $\lambda \geq 420$ $\mathrm{nm}$. The results indicate that mixture of CdS and Te has the lowest photocatalytic $\mathrm{H}_{2}$ production activity, likely because Te alone is not suitable for $\mathrm{H}_{2}$ evolution as a result of its low bandgap energy. Meanwhile, its presence may physically block CdS from absorbing light. However, CdS-Te heterostructures show significantly improved catalytic activity.

Figure $7 \mathrm{~b}$ compares the photocatalytic $\mathrm{H}_{2}$ production rates of CdS-Te, NiS/CdS-Te, $\mathrm{MoS}_{2} / \mathrm{CdS}-\mathrm{Te}$, and CdS-Te@Pt,Pd 
samples. Their compositions are listed in Table S1 in the Supporting Information. SEM images of CdS, Te, and physically mixed CdS/Te are presented in Figure S21. Notably, the results indicate that the $\mathrm{NiS} / \mathrm{CdS}-\mathrm{Te}$ heterostructure exhibits photocatalytic activity comparable to that of the CdSTe@Pt,Pd heterostructure. The apparent quantum efficiency of the NiS/CdS-Te catalyst was calculated to be $6.9 \%$. The photocatalytic activity and stability of $\mathrm{NiS} / \mathrm{CdS}$-Te relative to other CdS-based catalysts are summarized in Table S2. The overall activity of the $\mathrm{NiS} / \mathrm{CdS}$-Te heterostructure appears to be lower than that of the material prepared by $\mathrm{Xu}$ and coworkers, ${ }^{20}$ likely as a result of the low specific surface area $(21.3$ $\mathrm{m}^{2} / \mathrm{g}$; see Figure S22). Under visible-light irradiation, the electrons (e) in the valence band (VB) of $\mathrm{CdS}$ are excited to the conduction band $(\mathrm{CB})$, leaving behind holes $\left(\mathrm{h}^{+}\right)$in the VB. In general, these charge carriers may quickly recombine, and only a very small fraction of the electrons and holes are involved in the photocatalytic reaction process. ${ }^{13 a, 25,26}$ Therefore, pure CdS exhibits low photocatalytic activity. However, in the presence of conductive Te NTs, the separation of photogenerated electrons and holes is efficiently promoted. The photogenerated electrons in the CdS are transported on the surfaces of the Te NTs because of the large work function of the latter $(4.95 \mathrm{eV}),{ }^{27}$ as illustrated in Scheme 1. As a result, photocatalytic $\mathrm{H}_{2}$ production is greatly enhanced. Meanwhile, the holes are mainly consumed by lactic acid to generate $\mathrm{CO}_{2}$.

Scheme 1. Schematic Illustration of Charge Separation and Transfer in the CdS-Te Heterostructure Catalyst under Visible-Light Illumination

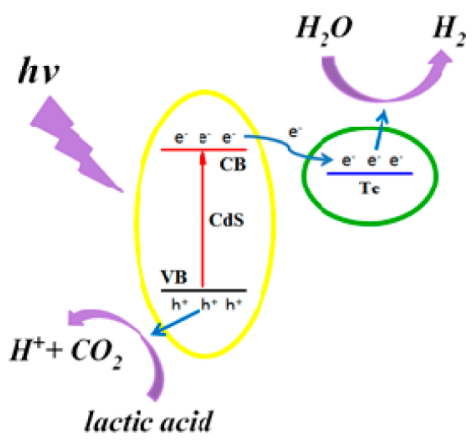

The additional loading of $\mathrm{NiS}, \mathrm{MoS}_{2}, \mathrm{Pt}$, and Pd cocatalysts onto the CdS-Te heterostructure provides more active sites for the surface reaction, leading to a significant enhancement of the $\mathrm{H}_{2}$ production (Figure 7 and Figure S23). The underlying photocatalytic mechanisms of the NiS/CdS-Te catalysts are schematically illustrated in Figure S24. Here, when p-type NiS nanoparticles (NPs) are combined with n-type CdS NPs, a number of $\mathrm{p}-\mathrm{n}$ junctions form at the interface. ${ }^{28}$ Because of the different Fermi levels of p-type NiS and n-type CdS, electrons in the n-type CdS fill some of the holes in the p-type NiS, as these holes are available at lower-energy states. This results in positively charged holes in the n-type CdS and extra negatively charged electrons in the p-type NiS. As a result, an internal electric field is formed at equilibrium. ${ }^{28}$ Driven by this internal field, the photogenerated holes in the VB of CdS are excited toward the $\mathrm{VB}$ of $\mathrm{NiS}$, and electrons are accelerated from the $\mathrm{CB}$ of $\mathrm{NiS}$ into the $\mathrm{CB}$ of $\mathrm{CdS}$ and then from the $\mathrm{CB}$ of $\mathrm{CdS}$ into $\mathrm{Te}$ in the NiS/CdS-Te heterostructure. The photogenerated electron-hole pairs are effectively separated by the $\mathrm{p}-\mathrm{n}$ junction, and a high concentration of electrons is thus obtained on the surface of Te. Therefore, the NiS/CdS-Te catalysts exhibit better photocatalytic activity for water splitting.

Under illumination, $\mathrm{S}^{2-}$ is more susceptible to oxidation than water, leading to the instability of metal sulfide photocatalysts. Figure 8 suggests that for the NiS/CdS-Te catalyst there is no

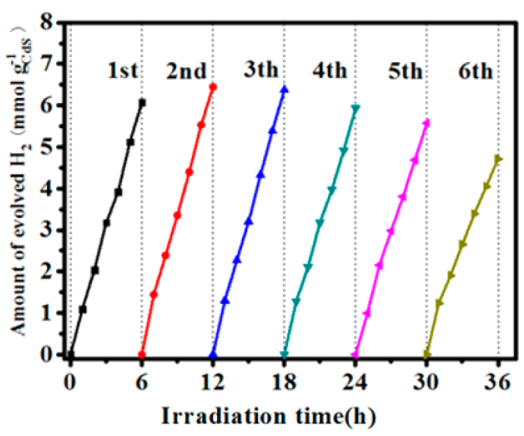

Figure 8. Recyclability of the NiS/CdS-Te catalyst in the $\mathrm{H}_{2}$ evolution reaction. The photocatalytic $\mathrm{H}_{2}$ evolution over $\mathrm{NiS} / \mathrm{CdS}-\mathrm{Te}$ was continued for $36 \mathrm{~h}$ with evacuation every $6 \mathrm{~h}$ (dotted vertical lines).

great change in the catalytic activity after $30 \mathrm{~h}$, signifying a marked improvement in the photostability over the existing MC catalysts. An SEM image of NiS/CdS-Te after $30 \mathrm{~h}$ of photoreaction (Figure S25) shows that the shape of the NiS/ CdS-Te catalyst was almost intact. The better photostability of the NiS/CdS-Te heterostructure over existing $\mathrm{NiS} / \mathrm{CdS}$ catalysts may be attributed to the presence of Te. On the other hand, a small quantity of CdTe produced at the interface of the Te NTs with the CdS spheres would form a CdS/CdTe solid solution with $\mathrm{CdS},{ }^{29}$ which should improve the photostability of CdS as well.

\section{CONCLUSIONS}

A simple and versatile strategy for crafting CdS-Te and CdSTe-based heterostructures was developed. This new synthetic strategy has a number of unique advantages. First, the experiments are convenient to perform, and the reaction conditions are mild. Second, the preparation depends on only two precursors, which reduces the cost. Finally, a series of CdS$\mathrm{Te} @ \mathrm{M} x \mathrm{Te}(\mathrm{M}=$ transition metal, e.g., Ag, Pb, Cd, Zn, etc.; $x=$ the number of Te atoms), CdS-Te@M, or CdS-Te@M,N (M and $\mathrm{N}$ refer to different noble metals, e.g., $\mathrm{Pt}$ and $\mathrm{Pd}$ ) heterostructures can be controllably created by utilizing Te as the self-sacrificial reagent.

Because of the unique chemical and physical properties of $\mathrm{Te}$, together with the efficient function of the cocatalyst, the resulting CdS-Te and CdS-Te-based composites exhibited enhanced photocatalytic activity and photostability for water splitting into $\mathrm{H}_{2}$. Moreover, the present obtained products could be expected to be applied in many other fields, such as solar cells, photodetectord, and photoelectronic devices.

\section{ASSOCIATED CONTENT}

\section{S Supporting Information}

The Supporting Information is available free of charge on the ACS Publications website at DOI: 10.1021/jacs.5b04784.

Detailed experimental procedures, Tables S1 and S2, and Figures S1-S25 (PDF) 


\section{AUTHOR INFORMATION}

\section{Corresponding Authors}

*shunwang@wzu.edu.cn

*zhiqun.lin@mse.gatech.edu

*jwang@uwindsor.ca

\section{Notes}

The authors declare no competing financial interest.

\section{ACKNOWLEDGMENTS}

We are grateful for financial support from the National Natural Science Foundation of China (51272182, 21471116, and 21301130), the Zhejiang Provincial Natural Science Foundation of China (LY13E020008 and Z15E020005), and the Zhejiang Scientific and Technological Innovation Fund (2013R424067).

\section{REFERENCES}

(1) (a) Gao, M. R.; Xu, Y. F.; Jiang, J.; Yu, S. H. Chem. Soc. Rev. 2013 42, 2986. (b) Chen, Z.; Higgins, D.; Yu, A.; Zhang, L.; Zhang, J. Energy Environ. Sci. 2011, 4, 3167. (c) Zheng, N.; Bu, X.; Feng, P. Nature 2003, 426, 428.

(2) (a) Spanhel, L.; Weller, H.; Henglein, A. J. Am. Chem. Soc. 1987, 109, 6632. (b) Zhang, J.; Zhu, Z. P.; Tang, Y. P.; Mullen, K.; Feng, X. L. Adv. Mater. 2014, 26, 734.

(3) (a) Wu, K. F.; Zhu, H. M.; Liu, Z.; Rodríguez-Córdoba, W.; Lian, T. Q. J. Am. Chem. Soc. 2012, 134, 10337. (b) Wu, K. F.; Chen, Z. Y.; Lv, H. J.; Zhu, H. M.; Hill, C. L.; Lian, T. Q. J. Am. Chem. Soc. 2014, 136, 7708. (c) Franchini, I. R.; Bertoni, G.; Falqui, A.; Giannini, C.; Wang, L. W.; Manna, L. J. Mater. Chem. 2010, 20, 1357.

(4) (a) Wang, W.; Lu, X. L.; Zhang, T.; Zhang, G. Q.; Jiang, W. J.; Li, X. G. J. Am. Chem. Soc. 2007, 129, 6702. (b) Zhang, Y. C.; Wang, H.; Kräemer, S.; Shi, Y. F.; Zhang, F.; Snedaker, M.; Ding, K. L.; Moskovits, M.; Snyder, G. J.; Stucky, G. D. ACS Nano 2011, 5, 3158.

(5) (a) Li, J. T.; Cushing, S. K.; Zheng, P.; Senty, T.; Meng, F. K.; Bristow, A. D.; Manivannan, A.; Wu, N. Q. J. Am. Chem. Soc. 2014, 136, 8438. (b) Zou, X. X.; Wang, P. P.; Li, C. J.; Zhao, J.; Wang, D. J.; Asefa, T.; Li, G. D. J. Mater. Chem. A 2014, 2, 4682.

(6) Meng, F. K.; Li, J. T.; Cushing, S. K.; Zhi, M. J.; Wu, N. Q. J. Am. Chem. Soc. 2013, 135, 10286.

(7) (a) Jing, L. J.; Kershaw, S. V.; Kipp, T.; Kalytchuk, S.; Ding, K.; Zeng, J. F.; Jiao, M. X.; Sun, X. Y.; Mews, A.; Rogach, A. L.; Gao, M.Y. J. Am. Chem. Soc. 2015, 137, 2073. (b) van Embden, J.; Jasieniak, J.; Mulvaney, P. J. Am. Chem. Soc. 2009, 131, 14299.

(8) (a) Gautam, U. K.; Fang, X. S.; Bando, Y.; Zhan, J. H.; Golberg, D. ACS Nano 2008, 2, 1015. (b) Zhu, W.; Liu, X.; Liu, H. Q.; Tong, D. L.; Yang, J. Y.; Peng, J. Y. J. Am. Chem. Soc. 2010, 132, 12619. (c) Wang, T.; Zhuang, J. Q.; Lynch, J.; Chen, O.; Wang, Z. L.; Wang, X. R.; LaMontagne, D.; Wu, H. M.; Wang, Z. G.; Cao, Y. C. Science 2012, 338, 358. (d) Ardalan, P.; Brennan, T. P.; Lee, H. B. R.; Bakke, J. R.; Ding, I. K.; McGehee, M. D.; Bent, S. F. ACS Nano 2011, 5, 1495. (e) Xiao, F. X.; Miao, J.; Wang, H. Y.; Liu, B. J. Mater. Chem. A 2013, $1,12229$.

(9) (a) Hsu, Y. J.; Lu, S. Y. Chem. Commun. 2004, 2102. (b) Deka, S.; Miszta, K.; Dorfs, D.; Genovese, A.; Bertoni, G.; Manna, L. Nano Lett. 2010, 10, 3770 .

(10) (a) Mubeen, S.; Singh, N.; Lee, J.; Stucky, G. D.; Moskovits, M.; McFarland, E. W. Nano Lett. 2013, 13, 2110. (b) Zhang, Q. H.; Ai, X.; Wang, L. J.; Chang, Y. X.; Luo, W.; Jiang, W.; Chen, L. D. Adv. Funct. Mater. 2015, 25, 966.

(11) (a) Yang, J.; Ma, M. Z.; Sun, C. C.; Zhang, Y. F.; Huang, W.; Dong, X. C. J. Mater. Chem. A 2015, 3, 1258. (b) Xiang, Q. J.; Yu, J. G.; Jaroniec, M. J. Am. Chem. Soc. 2012, 134, 6575.

(12) (a) Qiao, H.; Yuan, J.; Xu, Z. Q.; Chen, C. Y.; Lin, S. H.; Wang, Y. S.; Song, J. C.; Liu, Y.; Khan, Q.; Hoh, H. Y.; Pan, C. X.; Li, S. J.; Bao, Q. L. ACS Nano 2015, 9, 1886. (b) Wang, Z. Q.; Wang, J.; Sham, T. K.; Yang, X. G. J. Phys. Chem. C 2012, 116, 10375.
(13) (a) Ran, J. R.; Zhang, J.; Yu, J. G.; Jaroniec, M.; Qiao, S. Z. Chem. Soc. Rev. 2014, 43, 7787. (b) Chen, X. B.; Li, C.; Gratzel, M.; Kostecki, R.; Mao, S. S. Chem. Soc. Rev. 2012, 41, 7909.

(14) Xu, W. H.; Song, J. M.; Sun, L.; Yang, J. L.; Hu, W. P.; Ji, Z. Y.; Yu, S. H. Small 2008, 4, 888.

(15) (a) Brigham, E. S.; Weisbecker, C. S.; Rudzinski, W. E.; Mallouk, T. E. Chem. Mater. 1996, 8, 2121. (b) Zhang, G. Q.; Fang, H. Y.; Yang, H. R.; Jauregui, L. A.; Chen, Y. P.; Wu, Y. Nano Lett. 2012, 12, 3627. (c) Zhao, Y. W.; Jin, H. L.; Zhou, H.; Lin, J. J.; Wang, S.; Wang, J. C. J. Phys. Chem. C 2012, 116, 7416. (d) Moon, G. D.; Ko, S.; Xia, Y.; Jeong, U. ACS Nano 2010, 4, 2307. (e) Qian, H. S.; Yu, S. H.; Luo, L. B.; Gong, J. Y.; Fei, L. F.; Liu, X. M. Chem. Mater. 2006, 18, 2102. (f) Sreeprasad, T. S.; Samal, A. K.; Pradeep, T. J. Phys. Chem. C 2009, $113,1727$.

(16) Xu, H.; Xu, Y.; Pang, X.; He, Y.; Jung, J.; Xia, H.; Lin, Z. Sci. Adv. 2015, 1, e1500025.

(17) (a) Mayers, B.; Xia, Y. N. Adv. Mater. 2002, 14, 279. (b) Guan, L.; Wang, S.; Gu, W.; Zhuang, J. X.; Jin, H. L.; Zhang, W. M.; Zhang, T.; Wang, J. C. Sens. Actuators, B 2014, 196, 321.

(18) (a) Zhang, N.; Liu, S. Q.; Fu, X. Z.; Xu, Y. J. J. Mater. Chem. 2012, 22, 5042. (b) Rengaraj, S.; Venkataraj, S.; Jee, S. H.; Kim, Y.; Tai, C.-W.; Repo, E.; Koistinen, A.; Ferancova, A.; Sillanpaa, M. Langmuir 2011, 27, 352.

(19) Liu, J. W.; Chen, F.; Zhang, M.; Qi, H.; Zhang, C. L.; Yu, S. H. Langmuir 2010, 26, 11372.

(20) Zhang, W.; Wang, Y. B.; Wang, Z.; Zhong, Z. Y.; Xu, R. Chem. Commun. 2010, 46, 7631.

(21) Jin, J.; Yu, J. G.; Liu, G.; Wong, P. K. J. Mater. Chem. A 2013, 1, 10927.

(22) Sahoo, R.; Roy, A.; Ray, C.; Mondal, C.; Negishi, Y.; Yusuf, S. M.; Pal, A.; Pal, T. J. Phys. Chem. C 2014, 118, 11485.

(23) (a) Ge, J. P.; Li, Y. D. Adv. Funct. Mater. 2004, 14, 157. (b) Jang, J. S.; Joshi, U. A.; Lee, J. S. J. Phys. Chem. C 2007, 111, 13280. (c) Zhuang, Z.; Lu, X.; Peng, Q.; Li, Y. J. Am. Chem. Soc. 2010, 132, 1819.

(24) Chen, J. Z.; Wu, X. J.; Yin, L. S.; Li, B.; Hong, X.; Fan, Z. X.; Chen, B.; Xue, C.; Zhang, H. Angew. Chem., Int. Ed. 2015, 54, 1210.

(25) Chang, K.; Mei, Z. W.; Wang, T.; Kang, Q.; Ouyang, S. X.; Ye, J. H. ACS Nano 2014, 8, 7078.

(26) $\mathrm{Hu}, \mathrm{Y}$. H. Angew. Chem., Int. Ed. 2012, 51, 12410.

(27) (a) Zhang, Y. C.; Snedaker, M. L.; Birkel, C. S.; Mubeen, S.; Ji, X. L.; Shi, Y. F.; Liu, D. Y.; Liu, X. N.; Moskovits, M.; Stucky, G. D. Nano Lett. 2012, 12, 1075. (b) Zhang, G. Q.; Fang, H. Y.; Yang, H. R.; Jauregui, L. A.; Chen, Y. P.; Wu, Y. Nano Lett. 2012, 12, 3627.

(28) Zhang, J.; Qiao, S. Z.; Qi, L. F.; Yu, J. G. Phys. Chem. Chem. Phys. 2013, 15, 12088.

(29) (a) Mirsagatov, S. A.; Leiderman, A. Y.; Aitbaev, B. U.; Makhmudov, M. A. Phys. Solid State 2009, 51, 2032. (b) Jing, L. H.; Kershaw, S. V.; Kipp, T.; Kalytchuk, S.; Ding, K.; Zeng, J. F.; Jiao, M. X.; Sun, X. Y.; Mews, A.; Rogach, A. L.; Gao, M. Y. J. Am. Chem. Soc. 2015, 137, 2073. 